Proceedings of the 2010 Winter Simulation Conference

B. Johansson, S. Jain, J. Montoya-Torres, J. Hugan, and E. Yücesan, eds.

\title{
CONTINGENT CAPITAL WITH DISCRETE CONVERSION FROM DEBT TO EQUITY
}

Paul Glasserman

Graduate School of Business

Columbia University

New York, NY, 10027, USA
Behzad Nouri

Industrial Eng. \& Operations Research Dept. Columbia University

New York, NY, 10027, USA

\begin{abstract}
We consider the problem of valuing contingent capital in the form of debt that converts to equity when a capital ratio falls below a threshold. With continuous monitoring of the conversion trigger and with asset value modeled as geometric Brownian motion, the value admits a closed-form expression. Here we focus on the case of a discretely monitored trigger and the simulation of three potential mechanisms for conversion in discrete time. We show how to use the continuous-time formulas as control variates through exact joint simulation of the discrete- and continuous-time processes. We then investigate continuity corrections to approximate discrete-time results using continuous-time formulas and compare results across alternative conversion mechanisms.
\end{abstract}

\section{INTRODUCTION}

In a simple but fundamental model of a firm, bankruptcy occurs when the value of the firm's assets fall below a threshold determined by the firm's debt. In the case of a bank, minimum capital requirements imposed by regulators are intended to reduce the likelihood that the threshold is breached. Recent proposals to enhance the stability of the financial system include calls for contingent capital, including debt that converts to equity as a bank reaches a critical capital ratio - a ratio of equity to asset value. In related work, we have derived closed-form expressions for valuing such convertible debt when asset value is modeled as geometric Brownian motion, assuming the capital ratio is monitored continuously and the conversion process takes place continuously. In practice, the accounting information needed to monitor a capital ratio may be available only periodically. Here we focus on the discretely monitored case for which no formulas are available and simulation becomes an attractive option.

The process of conversion from debt to equity is driven by the evolution of the firm's asset value — in particular, by its running minimum. In the discrete-time setting, asset value is observed only at fixed dates. A "fair" allocation of the firm's equity between prior shareholders and creditors who become shareholders through the conversion of debt to equity depends on what is assumed about the evolution of asset value between the observation dates. We examine three rules for the allocation in discrete time.

We then investigate two ways in which the continuous-time formulas we derived in Glasserman and Nouri (2010) can be applied to the discrete-time case. First, we show how the continuous-time case can be used as a control variate for the discrete-time problems. This requires exact simulation of the continuous-time model together with the discrete-time model. Perhaps surprisingly, the continuous-time case is actually easier to simulate than the discrete-time case.

Then we examine continuity corrections in order to approximate discrete-time results using continuous-time formulas. For the running minimum, the correction of Asmussen, Glynn, and Pitman (1995) is highly effective. However, the allocation of equity between the original and converted shareholders turns out to be a more complex process. In the continuous-time case, we showed in Glasserman and Nouri (2010) that the allocation depends only on the running minimum of the asset value process. But in discrete time the allocation depends on the increments of the running minimum as well. We compare the effectiveness of continuity corrections across the three allocation rules and numerically investigate the joint distribution of the discrete and continuous allocation fractions.

Section 2 reviews the continuous setting of Glasserman and Nouri (2010) and Section 3 presents the discrete-time counterpart. Section 4 develops the control variate method. Section 5 introduces and tests the continuity corrections. 


\section{CONTINUOUS CONVERSION}

As in Glasserman and Nouri (2010), we consider contingent capital that automatically converts into common equity if the issuer's capital ratio (ratio of equity to assets) falls bellow a pre-specified threshold. The critical capital ratio (e.g., 5\%) is measured in terms of the "book" or accounting value of equity, not the market value. (Flannery (2005) and Sundaresan and Wang (2010) consider market-based triggers.) If we let $V_{t}$ denote the value of the firm's assets at time $t, B_{t}$ the remaining amount of contingent capital, and $D_{t}$ the amount of regular debt, then the firm's equity capital is $V_{t}-B_{t}-D_{t}$. A capital requirement specifying that equity must be at least a fraction of asset value then takes the form

$$
V_{t}-B_{t}-D_{t} \geq V_{t}
$$

or simply $V_{t} \geq\left(B_{t}+D_{t}\right) /(1-)$. A loss in value $V_{t}$ triggers the conversion of just enough contingent capital $B_{t}$ to maintain this bound, if posssible.

We apply a conversion ratio of $q$ in terms of book values, meaning that one dollar of debt converts into $q$ dollars of equity as soon as the trigger is tripped. This would imply that if $q>1$ there is a transfer of wealth from equity holders to debt holders at conversion which is achieved by the number of shares the debt holders receive. We will suppose that the convertible debt has principal value $B$ and coupon rate $c_{1}$, and the regular debt has principal value $D$ and coupon rate $c_{2}$. In addition we assume that both the convertible debt and the regular debt have maturity $T$, sell at par at issuance time and their corresponding coupon rates are such that they have constant book value.

In Glasserman and Nouri (2010), we assume that the conversion trigger is monitored continuously. We denote the firm's asset value by $V$ and model its risk-neutral dynamics as a geometric Brownian motion,

$$
\frac{d V_{t}}{V_{t}}=(r-) d t+d W_{t}
$$

where $r$ is the risk-free rate of interest, is the constant fraction of value paid out to security holders and $W_{t}$ is a standard Brownian motion. The conversion process depends on two boundaries

$$
a=\frac{B+D}{1-}, \quad b=\frac{D}{1-} ;
$$

conversion begins when $V_{t}$ reaches $a$, and the convertible debt is exhausted when $V_{t}$ reaches $b$. The amount of debt converted up to time $t$ is $(1-) L_{t}$, where

$$
L_{t}=\min \left\{\left(a-\min _{0 \leq s \leq t} V_{s}\right)^{+}, a-b\right\}
$$

is a function of the running minimum of $V$.

A critical step in valuing the contingent capital is determining the fraction of equity held by the original shareholders, as opposed to the investors who became shareholders as a result of the conversion of debt to equity. In Glasserman and Nouri (2010), we show that the proportion of the equity at time $t$ which belongs to the initial equity holders is

$$
t=\left(\frac{a-L_{t}}{a}\right)^{\left(q \frac{1-}{2}\right)} .
$$

In particular, the allocation is a function of the running minimum of the asset value process.

Defining $\equiv r-\quad-2 / 2$, we have

$$
V_{t}=V_{0} \exp \left\{t+W_{t}\right\}
$$

If we set

$$
\widetilde{W}_{t} \equiv \log \left(\frac{V_{t}}{V_{0}}\right)=t+W_{t}, \quad \widetilde{m}_{t} \equiv \min _{0 \leq u \leq t} \widetilde{W}_{t}
$$

then $\widetilde{W}_{t}$ has a $N\left(t,{ }^{2} t\right)$ distribution and $\left(\widetilde{W}_{t}, \widetilde{m}_{t}\right)$ has joint density

$$
f_{\widetilde{W}_{t}, \widetilde{m}_{t}}(w, m)=\frac{2(w-2 m)}{3 \sqrt{2 t^{3}}} \exp \left\{\frac{1}{2}\left(w-\frac{1}{2}{ }^{2} t-\frac{(w-2 m)^{2}}{2 t}\right)\right\}
$$




\section{Glasserman and Nouri}

for $m \leq 0$ and $w \geq m$. Conditional on the value of $\widetilde{W}_{t}$ we have a Brownian bridge connecting point zero to $\widetilde{W}_{t}$. The conditional density of the minimum on this path is obtained by dividing the above joint density by the marginal density of $\widetilde{W}_{t}$, which after integrating results in the well-known result that

$$
\mathbb{P}\left(\widetilde{m}_{t} \leq m \mid \widetilde{W}_{t}=w\right)=\exp \left\{\frac{2 m(w-m)}{t^{2}}\right\}
$$

As observed by Asmussen et al. (1995), this distribution is readily inverted and allows exact sampling from the joint distribution of the terminal value of the Brownian motion and its minimum on an interval. In our setting, this yields the following algorithm for simulating the continuous process.

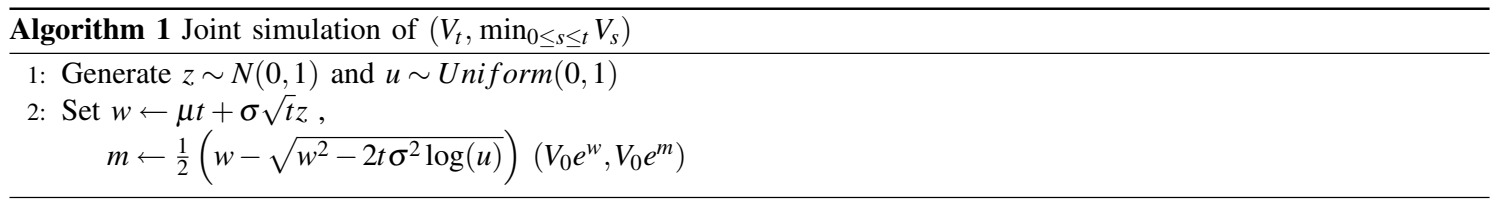

Given the terminal asset value and its minimum along the path, we can evaluate $L_{t}$ and ${ }_{t}$ through equations (1) and (2). Thus one can jointly simulate terminal value of the assets, the amount of debt converted into equity and the proportion of equity belonging to the initial stock owners in an efficient manner, without having to approximate the continuous-time process through a sequence of small time increments. In addition, the occurrence of a bankruptcy event defined as the assets' value hitting some constant value, i.e., $\mathbf{1}\left\{\min _{0 \leq s \leq t} V_{s} \leq b\right\}$, is a by-product of simulating $\min _{0 \leq s \leq t} V_{s}$.

\section{DISCRETE CONVERSION}

Now suppose that the capital ratio is monitored only at times $i t, i=0,1, \ldots, n$, where $t=T / n$. We write $\widehat{V}_{k}$ for $V_{k} t$, the assets' value at $k t$. The firm's book value of equity at the $k$ 'th monitoring time is $\widehat{V}_{k}-\left(B-(1-) \widehat{L}_{k}+D\right)$, where $\widehat{L}_{k}$ is the discrete version of $L_{t}$ defined by

$$
\widehat{L}_{k}=\min \left\{\left(a-\min _{0 \leq i \leq k} \widehat{V}_{i}\right)^{+}, a-b\right\}
$$

such that $(1-) \widehat{L}_{k}$ is the amount of convertible debt needed to be converted into equity in order to maintain the capital requirement at the monitoring points $i t, i=0,1, \ldots, k$.

The other important quantity is $\widehat{k}_{k}$, the fraction of equity belonging to the initial equity holders at the $k^{\prime}$ th monitoring time. The dynamics of ${ }_{k}$ depend on how the conversion mechanism is defined when asset value is observed periodically. We consider three possible mechanisms.

\subsection{Pure Discrete Conversion}

If between time $k t$ and $(k+1) t$ there is a loss in the firm's assets, equity holders pay for this loss (i.e. the equity of the firm loses value). If the loss is large enough to trigger conversion, then an amount $(1-) \widehat{L}_{k}$ of convertible debt (if available) converts to equity and boosts total equity to its minimum admissible level, (i.e., to a fraction of total assets). If the required capital is more than the available convertible debt, all the available convertible debt becomes equity, but, since the capital ratio remains under the threshold, the firm goes bankrupt and equity holders are wiped out.

Assuming sufficient contingent capital is available, a conversion ratio of $q$ requires a transfer of $(q-1)(1-) \widehat{L}_{k}$ dollars of value (in terms of book value) from stock owners to debt owners carried out through the number of new shares issued. If one dollar of debt is exchanged for $q$ dollars of equity, the difference would be a loss of $(q-1)$ dollars to the stock owners. Consequently, if there is conversion at $(k+1) t$, we would have

$$
\widehat{c}_{k+1}=\widehat{c}_{k} \times \max \{\overbrace{\widehat{V}_{k}-\left(B-(1-) \widehat{L}_{k}+D\right)+\widehat{V}_{k}}^{\text {equity at } k t-\overbrace{(q-1)(1-) \widehat{L}_{k}}^{t}-\text { loss in assets }}, 0\} / \overbrace{\left(\widehat{V}_{k}+\widehat{V}_{k}\right)}^{\text {transfer of wealth }} t
$$

The expression in the denominator in this equation is a valid assertion only if the loss in the assets does not wipe out the cushion provided by contingent capital. When equity is wiped out, the numerator becomes zero and the expression in the numerator remains valid. 

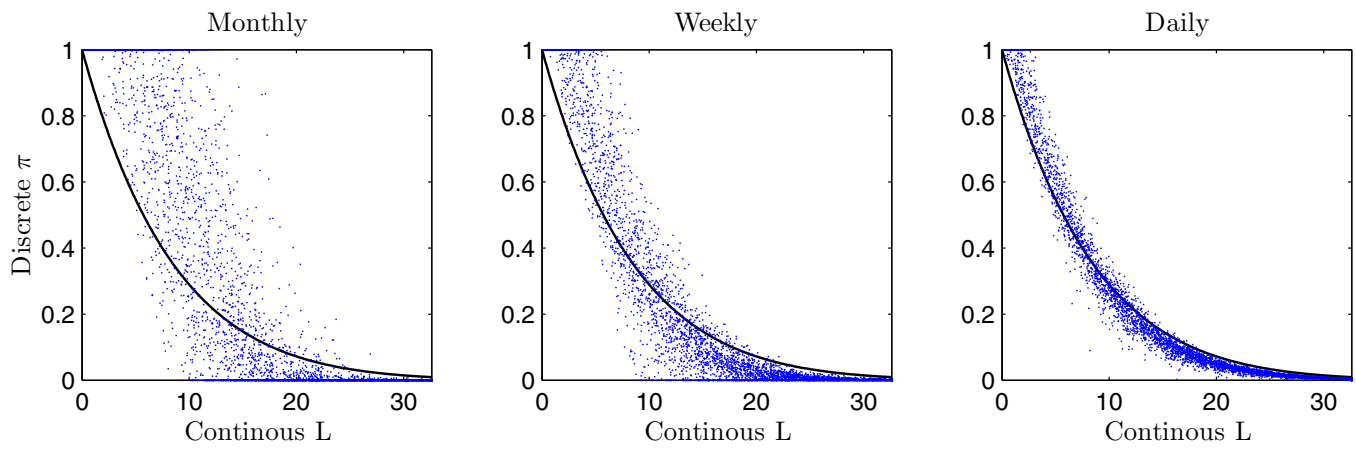

Figure 1: Discretely Computed $\widehat{ }_{n}$ versus Continuous $L_{T}$

If there is a conversion at time $k t$ then $\widehat{V}_{k}=\min _{0 \leq i \leq k} \widehat{V}_{i}$. This together with equation (3) allows us to derive from the above equation for $\widehat{k_{k}}$ that

$$
\left.\widehat{c}_{k+1}={ }_{i=1}^{k}\left(1-\min \left\{q \frac{1-}{a-\widehat{L}_{i+1}}\right), 1\right\}\right)
$$

This conversion mechanism is, in a sense, the most natural one in discrete time. It determines the amount of debt converted (and the resulting allocation of equity) based on the total change in asset value over the interval $t$. However, there is also a sense in which this mechanism is unfair. In implicitly assuming that the loss in asset value occurs all at once, it allocates a higher fraction of equity to converted shareholders because the prior shareholders bear the full cost of a sudden loss in asset value.

In the continuous case, we were able to express the amount of debt converted into equity based solely on the minimum value obtained by the asset value process between the issuance of debt and its maturity; conditional on the minimum value other properties of the path were irrelevant. Moreover, $T$ could be expressed as a deterministic function of the amount of debt converted into equity. However, when the capital ratio is monitored periodically, there is no deterministic relationship between $\widehat{ }_{n}$ and the amount of debt converted into equity (or equivalently $L_{t}$ ). Figure (1), shows the joint distribution of $\widehat{ }_{n}$ and $L_{t}$ for different monitoring frequencies. Each point in the plots corresponds to a sample path of asset value with discrete computation of ${ }_{n}$. The solid line represents the functional relationship between $T$ and $L_{T}$ in the continuous case (equation (2)). Increasing the monitoring frequency pushes the dots closer to the solid line, but the dots are widely dispersed at lower frequencies.

\subsection{Continuous Path Conversion}

A simple alternative to assuming that the loss in asset value occurs all at once would be to interpolate between $\widehat{V}_{k}$ and $\widehat{V}_{k+1}$ and then compute $\widehat{k}_{k+1} / \widehat{C}_{k}$ assuming that the conversion took place continuously along this path. Conditional on the end point values of a Brownian motion the most likely path would be a straight line, which suggests an exponential path for the geometric Brownian motion. Or, one could simply use linear interpolation between $\widehat{V}_{k}$ and $\widehat{V}_{k+1}$.

It may initially seem that to the number of possible paths connecting $\widehat{V}_{k}$ and $\widehat{V}_{k+1}$, one can get different values for

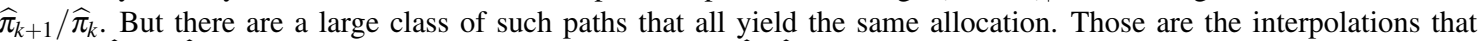
connect $\widehat{V}_{k}$ to $\widehat{V}_{k+1}$ through a continuous path above $\min \left\{\widehat{V}_{k}, \widehat{V}_{k+1}\right\}$. For example, this includes linear or exponential interpolation. For all such paths, the continuous formula for conversion (equation (2)) yields

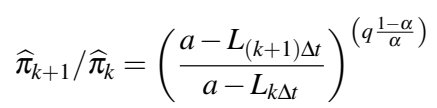

where $L_{k}{ }_{t}$ is computed based on the hypothetical path. However the fact that for $k t \leq t \leq(k+1) t, V_{t}$ is interpolated to some value above $\min \left\{\widehat{V}_{k}, \widehat{V}_{k+1}\right\}$ implies that $L_{k} t$ is equal to $\widehat{L}_{k}$. It follows that based on any such conversion mechanism

$$
\widehat{\widehat{c}}_{k}=\left(\frac{a-\widehat{L}_{k}}{a}\right)^{\left(q \frac{1-}{}\right)}
$$


Table 1: Variance reduction obtained by using the continuous case as control variate

\begin{tabular}{l|c|c|c||c|c|c} 
& $\operatorname{var}\left(\widehat{(}_{n}\right)$ & $\operatorname{var}\left(\widehat{n}_{n}-b^{*}\left({ }_{T}-\mathbf{E}\left[{ }_{T}\right]\right)\right)$ & Ratio & $\operatorname{var}\left(\widehat{L}_{n}\right)$ & $\operatorname{var}\left(\widehat{L}_{n}-b^{*}\left(L_{T}-\mathbf{E}\left[L_{T}\right]\right)\right)$ & Ratio \\
\hline Quarterly & 0.14592 & 0.04356 & 3 & 166.49 & 19.47 & 9 \\
Monthly & 0.10768 & 0.01656 & 7 & 145.67 & 6.353 & 23 \\
Weekly & 0.07837 & 0.00463 & 17 & 128.52 & 1.405 & 92 \\
Daily & 0.06531 & 0.00085 & 77 & 119.58 & 0.283 & 422
\end{tabular}

\subsection{Midpoint Conversion}

Alternatively, as an intermediate case between the previous two extremes of a single jump in asset value or a continuous decline, it is reasonable to consider a rule based on assuming that half the decline in asset value occurred midway through the interval and the remaining loss occurred at the end of the interval. Considering the interval $\left[\begin{array}{lll}k & t,(k+1) & t\end{array}\right]$, $(1-) \widehat{L}_{k}$ is the required conversion at the $k+1$ monitoring point. Breaking $\widehat{L}_{k}$ in half, and applying equation (4) we have

$$
\left.\widehat{\widehat{C}}_{k+1} / \widehat{\widehat{C}}_{k}=\left(1-\min \left\{q \frac{1-}{a-\widehat{L}_{k+1}+\widehat{L}_{k} / 2}\right), 1\right\}\right)\left(1-\min \left\{q-\left(\frac{1-\widehat{L}_{k} / 2}{a-\widehat{L}_{k+1}}\right), 1\right\}\right) .
$$

The simulation algorithm for all three discrete-time rules is detailed in Algorithm 2.

\section{USING THE CONTINUOUS CASE AS A CONTROL VARIATES}

The expected values of $T$ and $L_{T}$ can be computed in closed form, which makes these potentially attractive control variates for their discrete counterparts. However, to use them as controls, we need to generate the continuous minimum together with the discrete minimum. We cannot use Algorithm 1 for this purpose, because that algorithm ignores the intermediate values generated by Algorithm 2. Instead, over each step of the discrete path, we need to generate the conditional minimum given the endpoints of the interval; the minimum over the path is then the minimum over these intervals.

The steps are detailed in Algorithm 3. Using Algorithms 2 and 3 together, we can jointly simulate $\left({ }_{T}, L_{T}\right)$ and $\left(\widehat{ }_{n}, \widehat{L}_{n}\right)$. The resulting $\widehat{ }_{n}$ is highly correlated with $T$. Similarly $\widehat{L}_{n}$ and $L_{T}$ have a high correlation. Table (1) reports the resulting variance reduction. The values are reported for both $\widehat{\widehat{L}}_{n}$ and $\widehat{L}_{n}$ based on $10^{6}$ generated sample paths and pure discrete conversion mechanism. The parameters used are total assets $V_{0}=100$, convertible debt $B=30$, regular debt $D=60$, capital requirement ratio $=0.08$, risk-free interest rate $r=0.02$, volatility of assets $=0.36$, debt maturity $T=2$ and fractional payoff of the assets $=0.03$. The value $b^{*}$ for each calculation is set to maximize variance reduction. The results suggest that substantial variance reductions can be achieved. Moreover for higher monitoring frequencies the variance reduction is more effective which results from higher correlation between continuous and discrete quantities for smaller values of $t$.

\section{CONTINUITY CORRECTION}

Recall that in the discrete case, the capital ratio is monitored only at time points $i t, i=0,1, \ldots, n$, where $t=T / n$. Define $\widehat{W}_{k}$ as the $\log$ of asset value divided by $V_{0}$ at the $i$ 'th monitoring time, i.e. $\log \left(\widehat{V}_{k} / V_{0}\right)$, or equivalently $\widehat{W}_{k} \equiv \widetilde{W}_{k} t$. Thus, the $\widehat{W}_{i}$ 's are i.i.d with a normal distribution. In other words $\widehat{W}_{i}$ is a random walk with normal increments. The running minimum and hitting probabilities of such random walk can be approximated by their counterparts in the underlying continuous diffusion process, i.e. the running minimum and hitting probabilities of $\widetilde{W}_{t}$. Moreover one can apply a correction for the leading error term to get better estimates. More specifically define the running minimum of the random walk as $\widehat{m}_{n} \equiv \min _{0 \leq i \leq n} \widehat{W}_{i}$. Based on Asmussen et al. (1995) and consistent with the Siegmund (1985) corrected diffusion approximation for random walks, we have

$$
\mathbf{E}\left[e^{\widehat{m}_{n}}\right]=e^{\sqrt{t}} \mathbf{E}\left[e^{\widetilde{m}_{T}}\right]+o(1 / \sqrt{n})
$$

where

$$
=\lim _{n \rightarrow} \frac{\sqrt{n} \mathbf{E}\left[\widetilde{m}_{T}-\widehat{m}_{n}\right]}{\sqrt{T}} \approx 0.5826
$$

and $\widetilde{m}_{t}$ is the running minimum of the diffusion process, defined as $\min _{0 \leq u \leq t} \widetilde{W}_{t}$. 
Table 2: Continuity correction on $L_{t}$

\begin{tabular}{l|c|c|c|c|c} 
& $\mathbf{E}\left[L_{T}(a, b)\right]$ & $e$ & ${ }^{t} \mathbf{E}\left[L_{T}(\widehat{a}, \widehat{b})\right]$ & $\mathbf{E}\left[\widehat{L}_{n}(a, b)\right]$ & \multicolumn{2}{|c}{ Relative error (\%) } \\
& $(1)$ & $(2)$ & $(3)$ & $(3-1)$ & $(3-2)$ \\
\hline Quarterly & 24.67 & 20.69 & 20.79 & 18.68 & -0.47 \\
Monthly & 24.67 & 22.37 & 22.41 & 10.12 & -0.17 \\
Weekly & 24.67 & 23.57 & 23.58 & 4.65 & -0.05 \\
Daily & 24.67 & 24.17 & 24.18 & 2.05 & -0.02
\end{tabular}

This result can be applied to approximate the expected value of $\widehat{L}_{k}$ based on its continuous counterpart, $L_{t}$. Recall that $\widehat{L}_{k}$ is defined as $\left(a-\min _{0 \leq i \leq k} \widehat{V}_{i}\right)^{+} \wedge(a-b)$. We have

$$
\begin{aligned}
& \mathbf{E}\left[\widehat{L}_{n}\right]=\mathbf{E}\left[\left(a-V_{0} e^{\widehat{m}_{n}}\right)^{+} \wedge(a-b)\right] \\
& \approx \mathbf{E}\left[\left(a-V_{0} e^{\tilde{m}_{T}+} \sqrt{t}\right)^{+} \wedge(a-b)\right] \\
& =e^{\sqrt{t} \mathbf{E}}\left[\left(\widehat{a}-\min _{0 \leq u \leq T} V_{u}\right)^{+} \wedge(\widehat{a}-\widehat{b})\right]=e^{\sqrt{t}} \mathbf{E}\left[L_{T}(\widehat{a}, \widehat{b})\right]
\end{aligned}
$$

where $\widehat{a}$ and $\widehat{b}$ have replaced $a e^{-} \quad \sqrt{t}$ and $b e^{-} \quad \sqrt{t}$. This result is helpful as we have derived a closed-form expression for $\mathbf{E}\left[L_{T}\right]$.

Table (2) provides an example of the performance of this type of approximation. We consider different values of $T$, namely quarter, month, week and day. The last two columns of the table show the relative error of the continuous formula and the corrected formula in percent with respect to the true discretely computed value.

Continuity correction for $\widehat{{ }_{k}}$ is more subtle as it involves both the conversion mechanism and the difference between discretely observed values and their continuous counterparts. As a crude correction, one may just plug in the corrected $L_{t}$ into equation (2). Based on the results for continuity correction of $L_{t}$, we expect that this modification for would be a fairly good correction when the conversion method is based on hypothetical continuous paths as described in Section 3.2. More specifically if $\widehat{ }_{n}$ is as defined in equation (5) then

$$
\begin{aligned}
\mathbf{E}[\widehat{n}] & =\mathbf{E}\left[\left(\left(a-\widehat{L}_{n}\right) / a\right)^{\left(q \frac{1-}{2}\right)}\right] \\
& =\mathbf{E}\left[\left(\left(a-\left(a-V_{0} e^{\widehat{m}_{n}}\right)^{+} \wedge(a-b)\right) / a\right)^{\left(q \frac{1-}{-}\right)}\right] \\
& \approx \mathbf{E}\left[\left(\left(a-\left(a-V_{0} e^{\widetilde{m}_{T}+\sqrt{t}}\right)^{+} \wedge(a-b)\right) / a\right)^{\left(q \frac{1-}{}\right)}\right] \\
& =\mathbf{E}\left[\left(\left(\widehat{a}-L_{T}(\widehat{a}, \widehat{b}) / \widehat{a}\right)^{\left(q \frac{1-}{}\right)}\right]\right.
\end{aligned}
$$

To study how the continuously computed $T_{T}$ and discretely computed $\widehat{ }_{n}$ are related and how much the above continuity correction helps we apply the following algorithms. Algorithm (2) simulates a path step by step at each monitoring time point and computes $\widehat{k}_{k}$ and $\widehat{L}_{k}$ for $k=0,1, \cdots, n$. Based on the choice of conversion method the corresponding updating formula in step (5) should be applied.

Algorithm (3) takes the discretely simulated path by algorithm (2) as an input and conditional on the endpoint values of each interval simulates the minimum over that interval. The minimum of these values corresponds to the minimum of the entire path, and can be applied to compute continuous $L_{T}$ and ${ }_{T}$.

Figures (2) and (3) show the results. The plots correspond to monthly, weekly and daily conversion and the three different conversion methods discussed in Section 3. The $y$-axis is $\widehat{ }_{n}$. In Figure 2 the $x$-axis is the continuous $T$, whereas in Figure 3 the $x$-axis is the corrected $T$. In addition Table 3 provides the average values of $\widehat{n}_{n}$ under different conversion methods.

As can be observed from the figures, the bias and divergence from continuously computed ${ }_{T}$ is worst in the case of pure discrete conversion. For small values of $\widehat{ }_{n}$ there is an over estimation by the continuous $T$ while the bias is reversed for larger values. Applying the continuity correction to ${ }_{T}$ helps to have a more symmetric relationship for larger values of $\widehat{ }_{n}$ but also worsens the overestimation bias for a smaller values, resulting in a bigger error in the total average value when $t$ is small. This fact can also be observed in Table 3 .

For discrete conversion based on hypothetical continuous paths, as observed in the middle row of Figure 2, T is always a lower bound for $\widehat{ }_{n}$. This follows from the fact that in this case ${ }_{n}$ depend on the discretely observed 


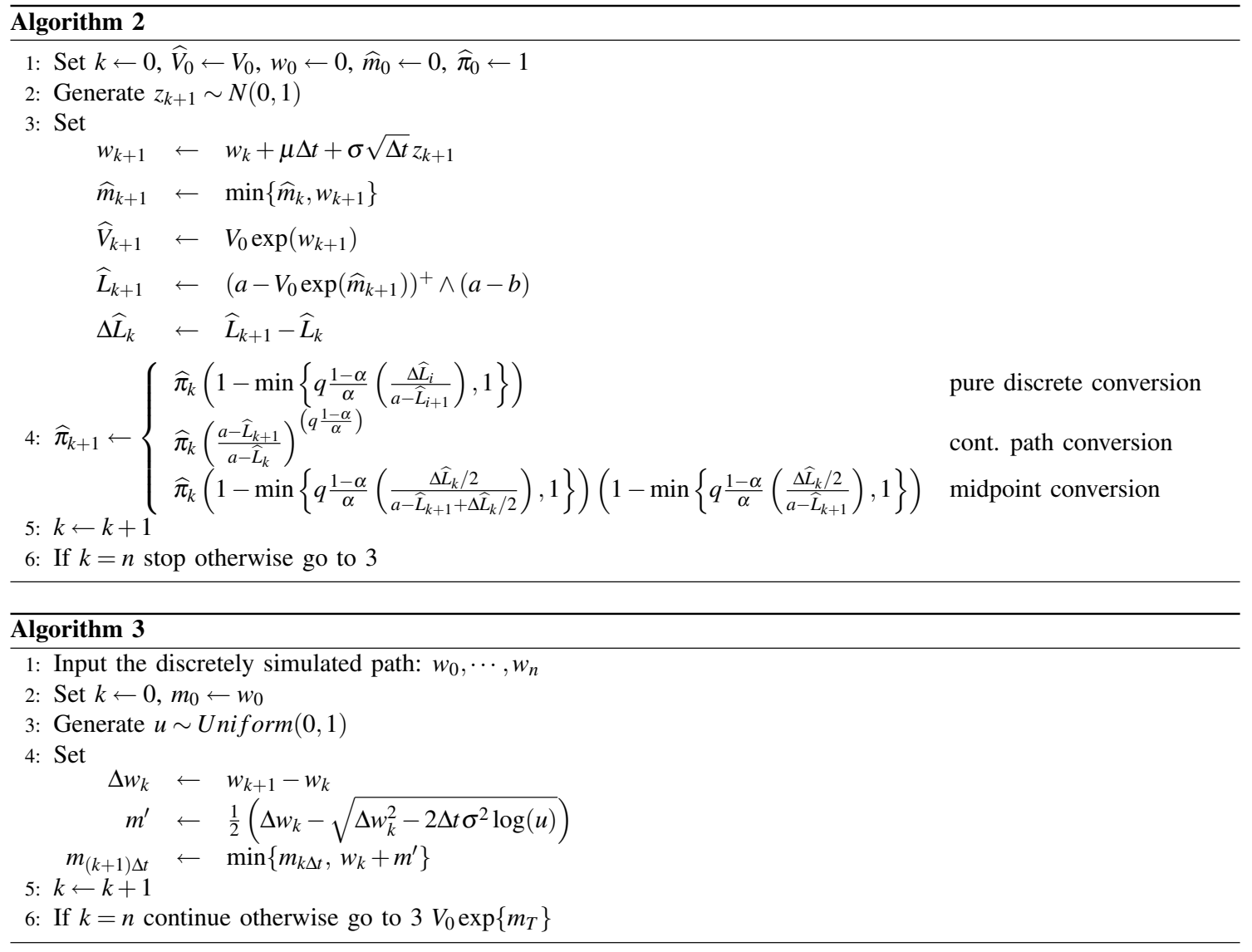

Table 3: Average from different conversion methods

\begin{tabular}{l|c|c|c|c} 
& Quarterly & Monthly & Weekly & Daily \\
\hline$\widehat{{ }^{n} \text { pure discrete }}$ & 0.204 & 0.151 & 0.123 & 0.122 \\
$\widehat{{ }^{n} \text { cont. path }}$ & 0.252 & 0.198 & 0.160 & 0.142 \\
${ }_{n}$ midpoint & 0.220 & 0.170 & 0.141 & 0.132 \\
$T(a, b)$ & 0.126 & 0.127 & 0.127 & 0.127 \\
$T(\widehat{a}, \widehat{b})$ & 0.236 & 0.189 & 0.156 & 0.140
\end{tabular}

minimum value of the path through the same functional relationship that ${ }_{T}$ has with continuously observed minimum of the path. It is clear that the discretely observed minimum is more than the continuous one, which confirms the observation that $\widehat{ }_{n}$ is larger than $T$. Applying the continuity correction to ${ }_{T}$ is considerably effective in making the distribution symmetric. In fact as shown in Table $3,{ }_{T}(\widehat{a}, \widehat{b})$ and discretely computed ${ }_{n}$ based on continuous paths are very close in the mean especially for smaller values of $t$.

As expected, the discretely computed $\widehat{ }_{n}$ based on the midpoint conversion rule, compared to the other two methods, has an intermediate joint distribution with the continuous $T$. This joint distribution is shown in the last rows of Figures 2 and 3. The overestimation bias for small values and underestimation bias for larger values is still present, though it is slightly mitigated compared to the pure discrete case. In addition the continuity correction on $T$ seems to be more helpful in making the relationship more symmetric.

\section{EARNED EQUITY THROUGH CONVERSION}

Part of the payoff to the owners of the convertible debt, comes from the value of equity that they earn through conversion. The discounted value of this payoff is given by

$$
e^{-r\left({ }_{b} \wedge n\right) t}\left(1-\widehat{(}_{\left({ }_{b} \wedge n\right)}\right)\left(\widehat{V}_{\left({ }_{b} \wedge n\right)}-\left[\left(B-(1-) \widehat{L}_{\left(\widehat{b}_{b} \wedge n\right)}\right)+D\right]\right)^{+}\left(1-\left(1-R_{1}\right) \mathbf{1}_{\left\{\widehat{b}_{b} \leq n\right\}}\right)
$$



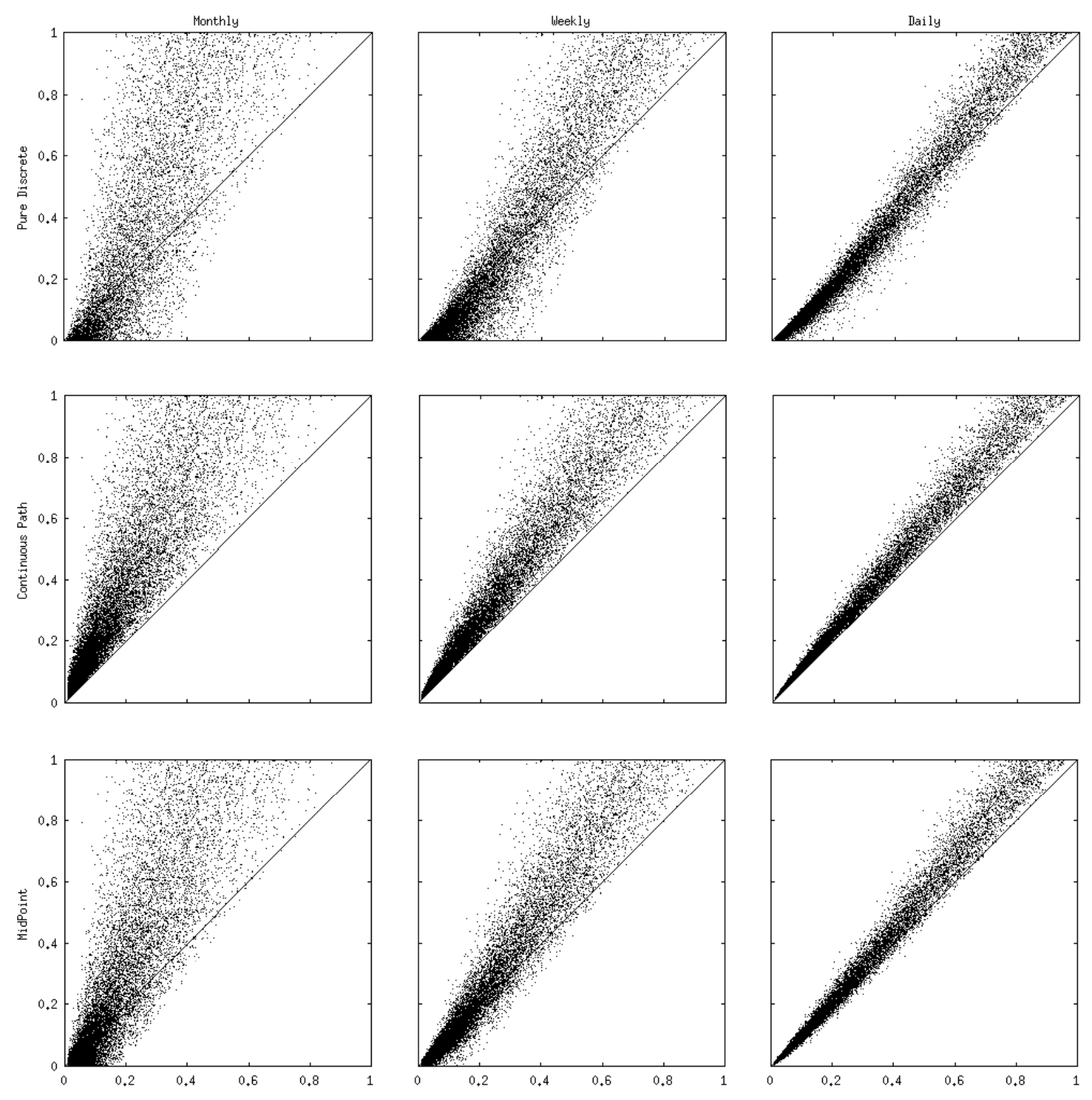

Figure 2: Illustration of the joint distribution of the discretely computed $\widehat{\widehat{n}}_{n}$ and the continuous $T$. The rows show alternative conversion rules, and the columns compare monitoring frequencies.

where $\widehat{b}_{b}$ is the first $k$ that $\widehat{V}_{k}$ is below the bankruptcy trigger $b,\left(\widehat{V}_{\left({ }_{b} \wedge n\right)}-\left[\left(B-(1-) \widehat{L}_{\left({ }_{b} \wedge n\right)}\right)+D\right]\right)^{+}$is the value of equity at either the debt maturity time or at liquidation time, and $R_{1}$ is the recovery rate on the equity in the case of liquidation or restructuring of the firm, representing the possible losses to the stock owners.

It would be of interest to see how the different conversion methods affect the above value. Table 4 provides such a comparison. As expected at each monitoring frequency, among discrete conversion methods, pure discrete conversion results in the largest value of equity allocated to owners of the convertible debt. This is in line with the fact that conversion of the debt into equity only at the end of the interval has the implicit assumption that the loss in asset value occurs all at once, and causes the prior shareholders to bear the full cost of the sudden loss in asset value, and therefore the converted shareholders get a higher fraction of the equity. Conversion based on continuous interpolation of asset value mitigates this situation, while midpoint conversion rule results in intermediate values.

We can also observe from Table 4 that increasing the monitoring frequency, increases the value of equity allocated to owners of convertible debt. This can partly be associated with the fact that monitoring capital ratio more frequently helps to catch low asset values more efficiently, resulting in more conversion of debt into equity. While if the interval between two monitoring times is large, the asset value can go well below the conversion threshold and then rise up again without any conversion taking place. The highest value for the equity owned by the converted stock owners takes 

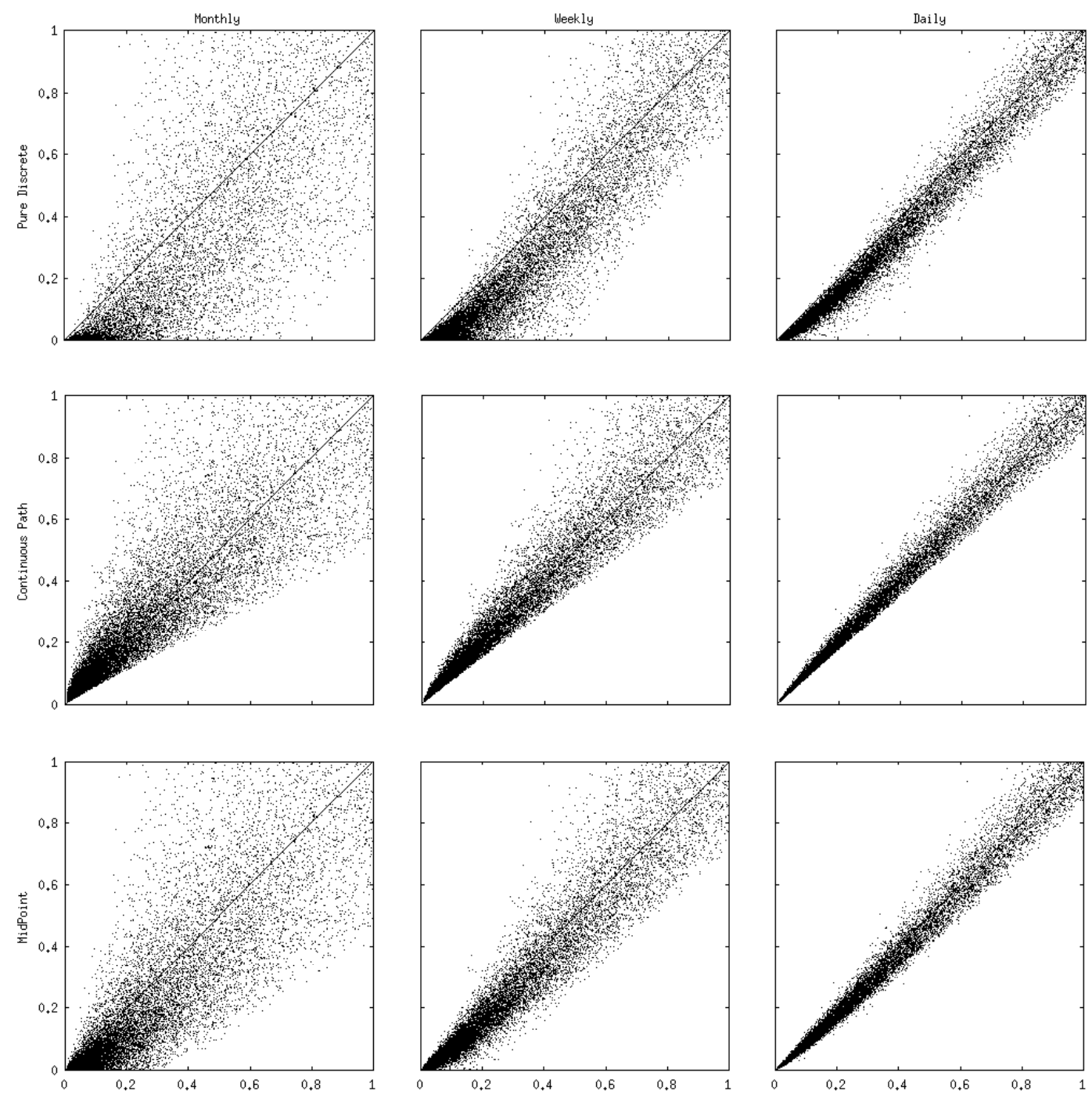

Figure 3: Illustration of the joint distribution of the discretely computed $\widehat{ }_{n}$ and the continuous $T$ with the continuity correction. The rows show alternative conversion rules, and the columns compare monitoring frequencies.

place in the limiting situation when capital ratio is monitored continuously in time. Applying continuity correction in this case results in values which are very close to the midpoint conversion rule in average values.

\section{CONCLUSION}

In comparison to the continuous case, the amount of debt converted into equity and the fraction of equity belonging to converted shareholders is more difficult to compute when the conversion happens discretely in time. In particular, the minimum of the asset value is no longer sufficient to determine $\widehat{ }_{n}$ and $\widehat{L}_{n}$. However the corresponding continuous values and equations are still useful at least in two ways. First, applying a continuity correction can help to obtain effective approximations for the amount of debt converted into equity. In addition, $\left({ }_{T}, L_{T}\right)$ can be used as control variates for $\left(\widehat{ }_{n}, \widehat{L}_{n}\right)$. The results show that the variance reduction obtained is substantial.

In discrete conversion of debt into equity, the conversion mechanism has an important effect on the sharing of losses between the initial shareholders and the owners of convertible debt. We compared three different potential methods that the conversion can be defined and in particular computed the expected value of the equity that will belong to owners 
Table 4: Earned equity through conversion

\begin{tabular}{l|c|c|c|c} 
& Quarterly & Monthly & Weekly & Daily \\
\hline Pure discrete conversion & 13.3 & 16.9 & 19.3 & 19.9 \\
Continuous hypothetical path & 11.5 & 14.8 & 17.6 & 19.0 \\
Midpoint conversion & 12.6 & 16.0 & 18.4 & 19.5 \\
Continuous conversion & 20.4 & 20.4 & 20.4 & 20.4 \\
Continuous conversion with correction & 13.0 & 15.9 & 18.2 & 19.4
\end{tabular}

of the convertible debt under each method. In addition, the effectiveness of the continuity correction also depends on how the discrete conversion is defined.

\section{ACKNOWLEDGMENTS}

This work is supported in part by NSF grant DMS0914539.

\section{REFERENCES}

Asmussen, S., P. Glynn, and J. Pitman. 1995, November. Discretization Error in Simulation of One-Dimensional Reflecting Brownian Motion. The Annals of Applied Probability 5 (4): 875-896.

Flannery, M. J. 2005. No Pain, No Gain? Effecting Market Discipline via 'Reverse Convertible Debentures'. In Capital Adequacy Beyond Basel: Banking Securities and Insurance, ed. H. S. Scott, Chapter 5. Oxford: Oxford University Press.

Glasserman, P., and B. Nouri. 2010. Pricing Contingent Capital with a Capital-Ratio Trigger. Working Paper.

Siegmund, D. 1985. Sequential analysis: Tests and confidence intervals. Berlin, Heidelberg, New York: Springer. Sundaresan, S., and Z. Wang. 2010. Design of Contingent Capital with Stock Price Trigger for Conversion.

\section{AUTHOR BIOGRAPHIES}

PAUL GLASSERMAN is the Jack R. Anderson Professor at Columbia Business School. His research focuses on modeling and computational problems in derivatives pricing and risk management. His book, Monte Carlo Methods in Financial Engineering (Springer, 2004), received the 2005 Outstanding Simulation Publication Award and the 2006 Lanchester Prize.

BEHZAD NOURI is a PhD student at Columbia University, Industrial Engineering and Operations Research Department. His research focuses on mathematical and computational finance. 